\title{
HOMMAGE
}

\section{Simone Veil}

\section{(13 juillet 1927 - 30 juin 2017 )}

\begin{abstract}
> Le 13 janvier 1975, inaugurant la célébration du $X^{\mathbf{e}}$ anniversaire de l'Inserm et les nouveaux locaux du siège de l'Institut rue de Tolbiac, Simone Veil, alors ministre de la Santé, a prononcé un discours très éclairant sur la finalité de la recherche médicale et sur le rôle d'une institution publique en la matière. En voici quelques extraits. <
\end{abstract}

«La recherche biomédicale est, dans une nation, le fondement indispensable d'une médecine de qualité. Le médecin ou le chirurgien ne peut se contenter d'être le lecteur-traducteur d'articles scientifiques étrangers, notamment en ce qui concerne les techniques de pointe. II sera d'autant plus apte à utiliser une nouvelle méthode thérapeutique qu'il aura contribué à la découvrir ou à la mettre au point dans le laboratoire de recherche avoisinant son département clinique. II s'ajoute à cela la vertu formatrice d'une activité de recherche pour un futur hospitalouniversitaire, qui acquerra par le travail de laboratoire les qualités de précision, de rigueur, de méthodologie si nécessaires dans un métier devenu de nature scientifique, sans perdre pour autant son caractère humain. Je ne vois d'ailleurs pas uniquement, dans la recherche biomédicale, le moyen d'améliorer qualitativement la médecine. En s'agrégeant les sciences humaines, la sociologie, la psychologie, l'étude des comportements, elle doit également permettre d'améliorer et de rationaliser le système de distribution des soins, préoccupation importante pour la santé publique.

Je tiens d'abord à rendre hommage à tous ceux qui ont permis d'obtenir de si remarquables résultats. Ce sont, en premier lieu, les chercheurs de toutes origines, les techniciens, les administratifs. Ce sont également, les personnalités qui ont participé à la gestion scientifique et administrative dans tous les conseils et commissions. À chacun d'eux va notre gratitude, [et je voudrais en particulier] saluer trois hommes qui ont particulièrement contribué à faire de l'Institut ce qu'il est devenu, les professeurs Bugnard, Aujaleu et Burg.

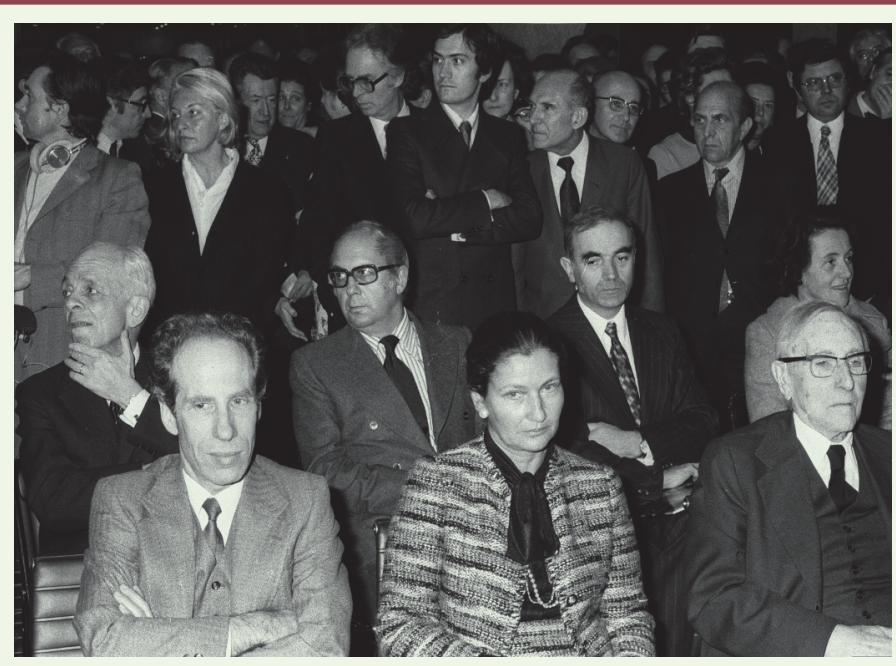

Au premier rang, Simone Veil et à sa gauche Robert Debré, l'instigateur de la grande réforme des études médicales (1958), réforme qui porte son nom (ㄷ) Inserm/Depardieu, Michel).

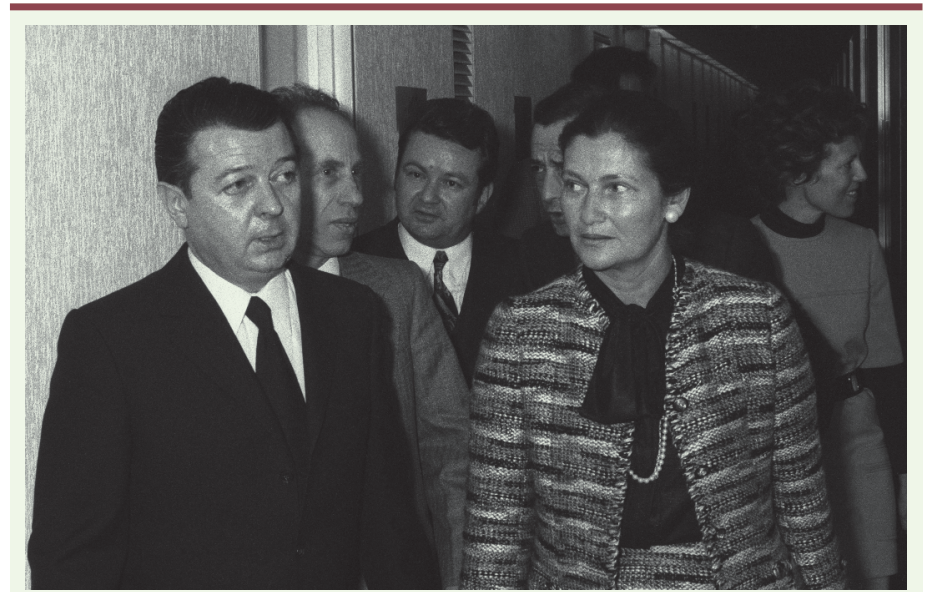

Constant Burg, directeur général de l'Inserm, et Simone Veil (๔ Inserm/ Depardieu, Michel).

Le développement de la recherche biomédicale [implique] d'être riche en hommes. C'est pourquoi, nous devons nous efforcer d'orienter vers cette branche les meilleurs de nos étudiants, venant de toutes les disciplines: médicales, biologiques, physiques, chimiques, électroniques, mathématiques, sociologiques, etc. L'un de nos objectifs doit être d'assurer aux 
équipes de chercheurs ayant fait sans conteste la preuve de leur efficacité et de leur créativité les crédits et les promotions qui soient à la mesure du service qu'elles rendent effectivement à la collectivité. En un tel domaine, les mots-clés doivent être souplesse et mobilité, sans que soient pour autant négligés le respect de la dignité des hommes ni le souci de leur sécurité professionnelle.

Ces chercheurs, ces équipes, cet Institut de la prochaine décennie, dans quelles directions, sur quels axes vont-ils orienter leurs efforts? Je souscris pleinement aux vœux du Professeur Jean Bernard qui souhaiterait voir privilégier la chimie du cerveau et les recherches portant sur la prévention.

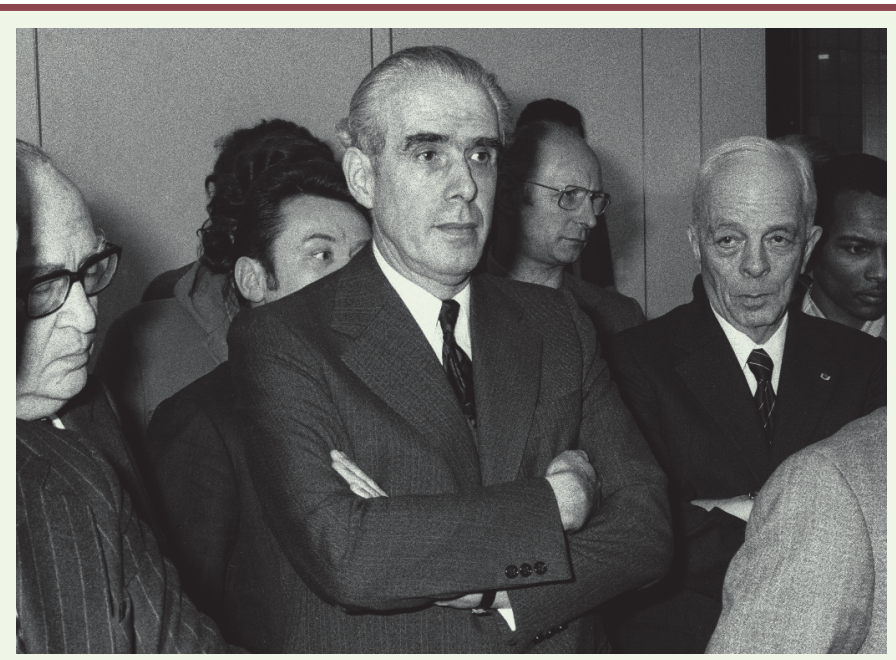

Pierre Royer, ancien président du conseil scientifique de I'Inserm, et Jean Bernard, ancien président du conseil d'administration de l'Inserm (ㄷ Inserm/ Depardieu, Michel).

[Mais il faut aussi] sauvegarder, j'insiste beaucoup sur ce point, un vaste secteur de recherche libre, non programmée, la terra incognita d'où viennent certaines des plus grandes découvertes du génie humain. Il est non moins évident que la collectivité, qui supporte financièrement la recherche, a le droit de porter son intérêt sur de grands problèmes qui, pour des raisons diverses, n'ont pas spontanément attiré l'attention des chercheurs. Le mot-clé me paraît être ici : équilibre.

La grande aventure de l'humanité s'est construite depuis les origines sur une séquence de découvertes ou de créations qui ont transformé la vie, tantôt de manière lente et progressive tantôt de manière brutale et rapide. La recherche biomédicale, dans son sens le plus étendu, c'est-à-dire débordant largement sur les sciences humaines, peut imprégner, influencer, orienter - en un mot, humaniser - tous les grands progrès scientifiques et préparer l'homme à son avenir. C'est I'honneur de l'Inserm, de ses chercheurs, de tous ceux qui l'animent et assurent son développement, que de servir ainsi l'un des idéaux les plus élevés de notre civilisation. » $\diamond$
> Le 12 octobre 1993, I'Inserm recevait Simone Veil, alors ministre d'État, ministre des Affaires sociales, de la Santé et de la Ville. Trois thèmes de discussion étaient à l'ordre du jour : la recherche en génétique humaine; le développement des relations entre l'Inserm, les universités, les CHU et leurs partenaires institutionnels de recherche; la recherche au service d'une politique de santé. <

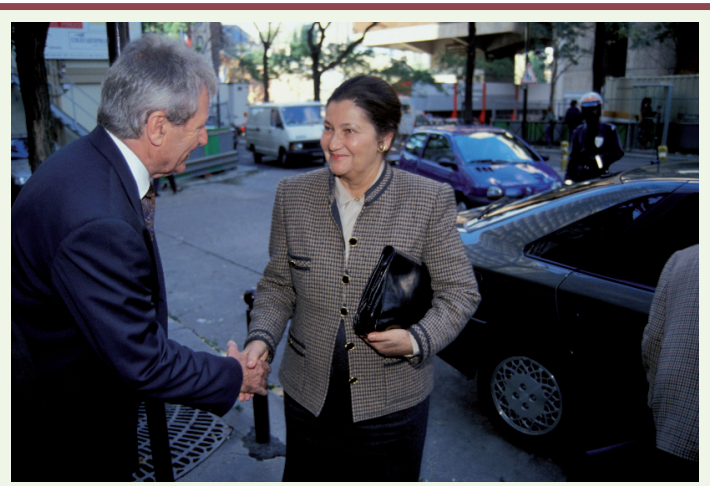

Accueil de Simone Veil, par André Kirn, président du conseil d'administration de l'Inserm (๔ Inserm/Depardieu, Michel).

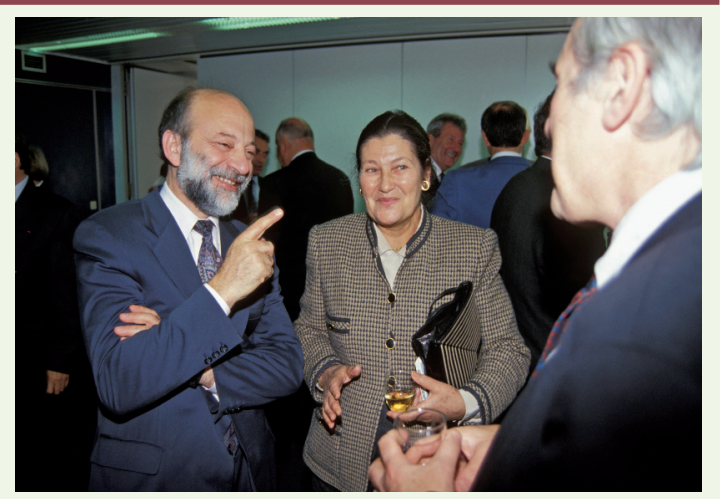

Simone Veil et Philippe Lazar, directeur général de l'Inserm (๔) Inserm/Depardieu, Michel).

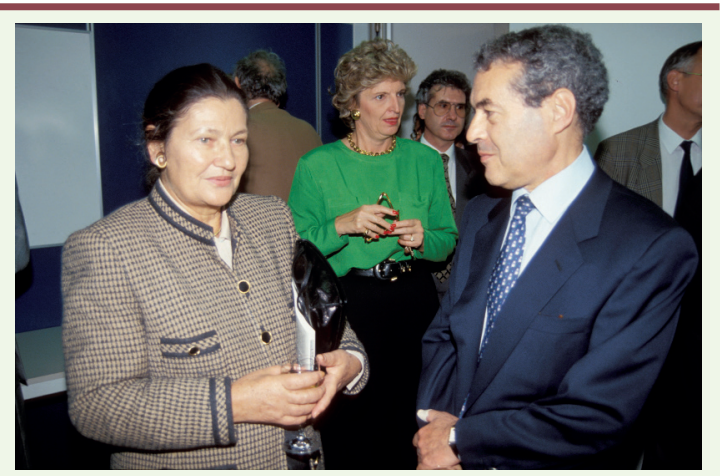

Simone Veil et Claude Amiel, président du conseil scientifique de I'Inserm. Au second rang, Christine Chirol, directrice du département des relations internationales ( Inserm/Depardieu, Michel). 